infarcts showed a degree of functional recovery after AMI. Volume and intensity of hyperenhancement on T2w CMR may give insights into functional recovery post reperfused AMI.

\section{PRE-CONTRAST T1 MAPPING ALLOWS ASSESSMENT OF SEVERITY OF ACUTE ISCHAEMIC MYOCARDIAL INJURY}

doi:10.1136/heartjnl-2012-301877b.90

${ }^{1} \mathrm{E}$ Dall'Armellina, ${ }^{1} \mathrm{~S}$ Piechnik, ${ }^{1} \mathrm{~V}$ M Ferreira, ${ }^{1} \mathrm{M} \mathrm{D}$ Robson, ${ }^{1} \mathrm{~J} \mathrm{M}$ Francis, ${ }^{2} \mathrm{~F}$ Cuculi, ${ }^{2} \mathrm{R}$ K Kharbanda, ${ }^{2} \mathrm{~A} P$ Banning, ${ }^{3} \mathrm{R}$ P Choudhury, ${ }^{1} \mathrm{~T}$ D Karamitsos, ${ }^{1} \mathrm{~S}$ Neubauer. ${ }^{1}$ OCMR Department of Cardiovascular Medicine, John Radcliffe Hospital, Oxford, UK; ${ }^{2}$ Department of Cardiology, John Radcliffe Hospital, Oxford, UK; ${ }^{3}$ Department of Cardiovascular Medicine, Oxford University, Oxford, UK

Introduction Cardiovascular magnetic resonance (CMR) is the gold standard technique to assess myocardial viability (using late gadolinium enhancement (LGE)) and reversible injury (using T2Weighted (T2W) for oedema imaging) in acute myocardial infarction (MI). However, both LGE and T2W are hampered by methodological issues such as threshold-based method for post-processing with scope for error and the need for MR contrast agent. The interpretation of CMR is also challenged by the dynamic changes occurring in the acutely ischaemic tissue as part of the healing process. Pre-contrast T1-mapping can overcome these limitations by providing voxel-based quantitative tissue characterisation. In acute MI patients, we sought to investigate whether pre-contrast T1mapping11 (1) detects acute myocardial injury, (2) allows for quantification of the severity of damage when compared to standard techniques such as LGE and T2W, and (3) has the ability to predict long term functional recovery.

Methods 41 patients with acute MI (30\% non-ST elevation MI (NSTEMI)) underwent 3T CMR including T2W, T1 mapping and LGE, $12-48 \mathrm{~h}$ after chest pain onset and at 6 months. Patients with ST elevation MI (STEMI) underwent primary PCI first. Acute mean segmental T1values, acute and chronic regional and global function and segmental damaged fraction by T2W and LGE were assessed.

Results The diagnostic performance of acute T1-mapping was at least as good as that of T2W CMR for detecting myocardial injury; however, in NSTEMI it was significantly higher than T2W oedema imaging. Also, T1 values could define the segmental damaged fraction, as assessed by either by LGE or T2W $(p<0.01)$. Furthermore, the likelihood of improvement of segmental function at 6 months decreased progressively as acute T1 values increased $(p<0.0004)$.

Conclusions In patients with acute MI, pre-contrast T1 mapping allows to delineate the extent of myocardial injury and to predict functional recovery at 6 months. Further investigations will be needed to determine whether T1 mapping can distinguish oedema from necrosis in acute MI.

\section{T1-MAPPING HAS A HIGH DIAGNOSTIC PERFORMANCE IN PATIENTS PRESENTING WITH ACUTE MYOCARDITIS: A CARDIOVASCULAR MAGNETIC RESONANCE STUDY}

doi:10.1136/heartjnl-2012-301877b.91

${ }^{1} \mathrm{~V} M$ Ferreira, ${ }^{1} \mathrm{~S} K$ K Piechnik, ${ }^{1} \mathrm{E}$ Dall'Armellina, ${ }^{1} \mathrm{~T} \mathrm{D}$ Karamitsos, ${ }^{1} \mathrm{~J} \mathrm{M}$ Francis, ${ }^{1} \mathrm{R}$ P Choudhury, ${ }^{2} \mathrm{~A}$ Kardos, ${ }^{3} \mathrm{M}$ G Friedrich, ${ }^{1} \mathrm{M} \mathrm{D}$ Robson, ${ }^{1} \mathrm{~S}$ Neubauer. ${ }^{1}$ University of Oxford, Oxford, UK; ${ }^{2}$ Milton Keynes NHS Hospital Foundation trust, Milton Keynes, UK; ${ }^{3}$ Université de Montréal, Montréal, Quebec, Canada

Background The diagnosis of acute myocarditis can be challenging. Cardiovascular magnetic resonance imaging (CMR) can be a useful tool in this setting but often requires multiple modalities for tissue characterisation, including T2-weighted (T2w), early and late gadolinium imaging. Cardiac T1-mapping is a novel technique that

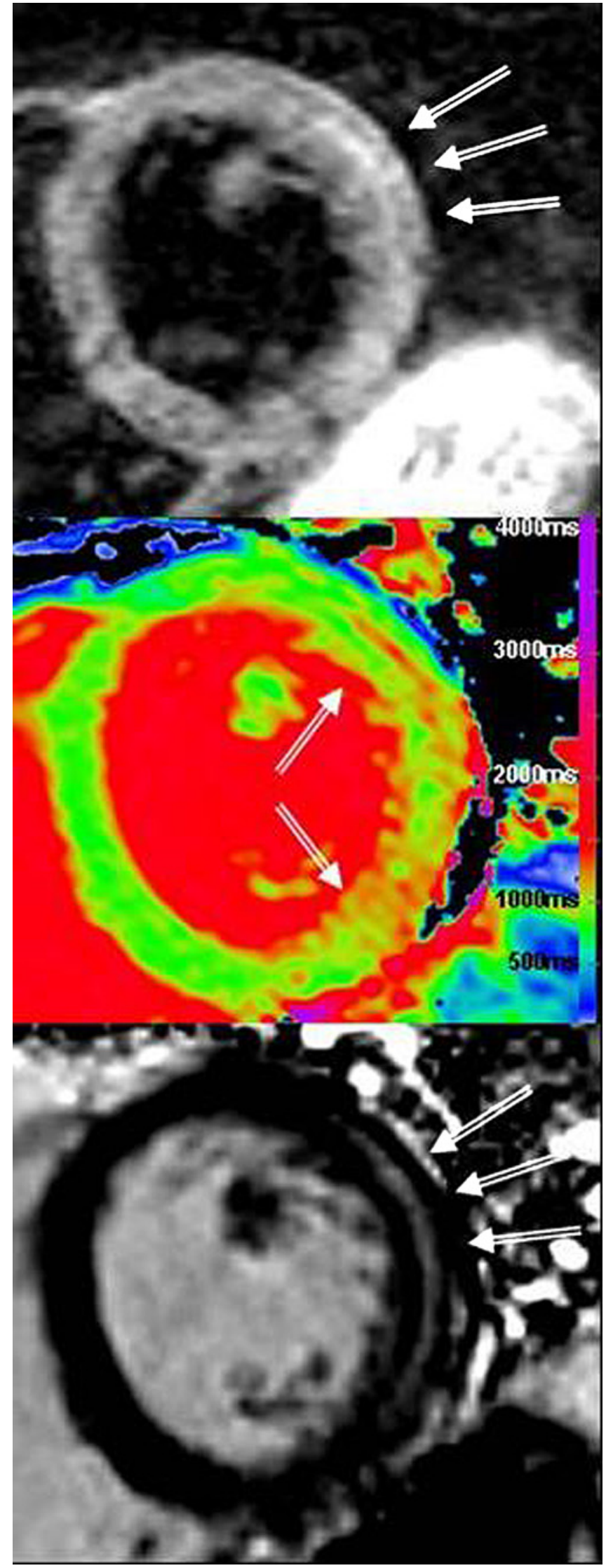

Abstract 091 Figure 1 Acute myocarditis. (Top) STIR demonstrating increased signal intensity in the mid lateral wall. (Middle) ShMOLLI T1map demonstrating increased T1 values $(1100-1200 \mathrm{~ms})$ in the lateral wall. (Bottom) LGE imaging demonstrating mid-wall enhancement in the lateral wall. 